\title{
Ternas pitagóricas suas relações e aplicações na Álgebra e na Geometria
}

\author{
Pythagoric terns its relationships and applications in Algebra and Geometry \\ Pitagórico tiene sus relaciones y aplicaciones en Álgebra y Geometría
}

Odirley Willians Miranda Saraiva

ORCID: https://orcid.org/0000-0001-9090-3788 Universidade Regional do Noroeste do Estado do Rio Grande do Sul, Brasil

Gustavo Nogueira Dias

ORCID: https://orcid.org/0000-0003-1315-9443

Colégio Federal Ten. Rêgo Barros Souza, Brasil

E-mail: gustavonogueiradias@gmail.com

Fabricio da Silva Lobato

ORCID: https://orcid.org/0000-0002-8240-8039 Universidade do Estado do Pará, Brasil

E-mail: fabriciolobatomat15@hotmail.com

Eldilene da Silva Barbosa

ORCID: https://orcid.org/0000-0002-9980-2286

Universidade Federal Rural da Amazônia, Brasil

E-mail: eldilene.barbosa@gmail.com

Gilberto Emanoel Reis Vogado

ORCID: https://orcid.org/0000-0003-4763-4767 Universidade do Estado do Pará, Brasil E-mail: gvogado@globo.com

Katiane Pereira da Silva

ORCID: https://orcid.org/0000-0001-7864-6467 Universidade Federal Rural da Amazônia, Brasil

E-mail: Katiane.silva@ufra.edu.br

Antonio Thiago Madeira Beirão

ORCID: https://orcid.org/0000-0003-1366-5995 Universidade Federal Rural da Amazônia, Brasil

E-mail: Thiago.madeira@ufra.edu

Nazaré Doriene de Melo Reis

ORCID: https://orcid.org/0000-0002-1711-8633

Faculdade Estácio do Pará, Brasil

E-mail: n.dmelo@hotmail.com

Reginaldo Pereira Flor

ORCID: https://orcid.org/0000-0003-4125-2434 Secretaria de Educação do Estado do Pará, Brasil

E-mail: reginaldopereiractrb@gmail.com

Herson Oliveira da Rocha

ORCID: https://orcid.org/0000-0002-2494-6277

Universidade Federal Rural, Brasil

E-mail: herson@ufra.edu.br

Cássio Pinho dos Reis

ORCID: https://orcid.org/0000-0002-2211-2295 Universidade Federal de Mato Grosso do Sul, Brasil

E-mail: cassio.reis@ufms.br

\begin{abstract}
Resumo
Este presente artigo tem o objetivo de mostrar uma nova forma de calcular as infinitas trincas pitagóricos relacionado como uma hipérbole equilátera, através de uma prova direta. Além de fornecer as aplicações na geometria plana e espacial, mostrando a agilidade do conhecimento e utilidade dos ternos pitagóricos, fazendo a relação de proporção com os lados do triângulo retângulo, apresentando dois tipos de ternas mais usuais no cálculo geométrico, trazendo com estes casos, quatro tipos usuais e importantes na geometria, o triângulo de lados: 3,4 e 5; 5,12 e 13; 8, 15 e 17 e o 7,24 e 25. A metodologia é uma investigação diagnóstica e abordagem (quantitativa), o estudo envolveu uma abordagem quantitativa que utiliza métodos quantitativos. Este artigo oferece dois casos importantes de obtenção de infinitas ternas, porém estes quatro casos resumem a maioria das aplicações discriminando assim, novas transposições didáticas importante para o ensino da matemática no contexto geral, facilitando o entendimento e diminuindo a complexidade dos cálculos.
\end{abstract}

Palavras-chave: Ternas pitagóricas; Hipérbole equilátera; Ensino da matemática. 


\begin{abstract}
This present article aims to show a new way to calculate the infinite Pythagorean cracks related as an equilateral hyperbola, through a direct proof. In addition to providing applications in plane and spatial geometry, showing the agility of knowledge and usefulness of the Pythagorean suits, making the relation of proportion with the sides of the right triangle, presenting two types of most common suits in geometric calculus, bringing with these cases, four usual and important types in geometry, the triangle of sides: 3,4 and 5; 5.12 and 13; 8, 15 and 17 and 7,24 and 25 . The methodology is a diagnostic investigation and approach (quantitative), the study involved a quantitative approach that uses quantitative methods. This article offers two important cases of obtaining infinite tenders, but these four cases summarize most of the applications, thus discriminating new didactic transpositions important for the teaching of mathematics in the general context, facilitating the understanding and reducing the complexity of calculations.
\end{abstract}

Keywords: Pythagoric terns; Equilateral hyperbole; Mathematics teaching.

\title{
Resumen
}

El presente artículo tiene como objetivo mostrar una nueva forma de calcular las infinitas fisuras pitagóricas relacionadas como hipérbola equilátera, a través de una demostración directa. Además de brindar aplicaciones en geometría plana y espacial, mostrando la agilidad de conocimiento y utilidad de los trajes pitagóricos, realizando la relación de proporción con los lados del triángulo rectángulo, presentando dos tipos de trajes más comunes en el cálculo geométrico, trayendo consigo estos casos, cuatro tipos habituales e importantes en geometría, el triángulo de lados: 3, 4 y 5; 5,12 y 13; 8, 15 y 17 y 7,24 y 25. La metodología es una investigación y enfoque diagnóstico (cuantitativo), el estudio involucró un enfoque cuantitativo que utiliza métodos cuantitativos. Este artículo ofrece dos casos importantes de obtención de ofertas infinitas, pero estos cuatro casos resumen la mayoría de las aplicaciones, discriminando así nuevas transposiciones didácticas importantes para la enseñanza de las matemáticas en el contexto general, facilitando la comprensión y reduciendo la complejidad de los cálculos.

Palabras clave: Licitaciones pitagóricas; Hipérbole equilátero; Enseñanza de las matemáticas.

\section{Introdução}

Os lados do triângulo retângulo, tem despertado a atenção de muitos matemáticos, engenheiros e profissionais das áreas, devido a sua exatidão ao se calcular qualquer um de seus lados e as suas aplicações na Geometria plana e espacial.

Segundo Maev Kennedy (2017), através dos séculos diversas gerações de estudiosos, cientistas e matemáticos têm tentado achar uma solução geral para o problema de números congruentes, encontrando, na maioria das vezes, soluções parciais. Uma solução geral implicaria encontrar um algoritmo que permitisse determinar quando um número natural é congruente ou não.

Na concepção de Secco, et al (2020), o ensino da matemática não é uma tarefa fácil, observa-se que existem algumas razões para esta dificuldade, talvez pela ausência de recursos pedagógicos e/ou falta de aplicação de uma metodologia de ensino que proporcione aos alunos o interesse em aprender, mais especificamente, falta de exemplos práticos que mostrem a aplicação da matemática no cotidiano dos alunos fora do ambiente escolar.

A Trigonometria - estudos dos triângulos - serviu desde os antigos babilônios, até pouco antes de Descartes, como instrumento puramente prático de agrimensura, astronomia e navegação. Os perscrutadores de estrelas e marinheiros, sondando os céus ou varrendo os mares, frequentemente necessitavam calcular distâncias que não podiam ser medidas com a régua ou a trena. A Trigonometria tornava isso possível pela simples aplicação de certas regras básicas sobre as relações entre os lados e os ângulos de qualquer triângulo, grande ou pequeno (Dias, G. N., 2011)

Presume-se que tenha sido Hiparco quem de fato estabeleceu, por volta de século II a.C., as bases da trigonometria. Mas os hindus e os árabes também contribuíram para seu desenvolvimento.

Contudo, foi na Europa do século XV que a trigonometria começou a ganhar importância, graças à influência de vários matemáticos, sobretudo o alemão Johann Müller, mais conhecido pelo nome latino Regiomontanus.

Regiomontanus trabalhou pela organização da trigonometria como uma disciplina independente da astronomia, além de escrever vários livros sobre tema, como de triangulis, em 1464. (Iezzi, 1993) 
O currículo da Educação Básica, particularmente o do Ensino Médio é regido pelos Parâmetros Curriculares Nacionais (PCN, Ensino Médio, 2002), com base nos princípios da Lei de Diretrizes e Bases da Educação Nacional (9.394/96, LDB). A esse respeito, reza o artigo 22 que:

A educação básica tem por finalidades desenvolver o educando, assegurar-lhe a formação comum indispensável para o exercício da cidadania e fornecer-lhe meios para progredir no trabalho e em estudos posteriores (Brasil, 1996, p. 22).

$\mathrm{Na}$ verdade, a ânsia do professor é tentar repassar os conteúdos de uma maneira bem fácil e acessível ao aluno, utilizando várias transposições didáticas que não são expostas nos livros didáticos e nem nos livros recomendados aos exames vestibulares. Neste sentido a contribuição de Chevallard (1986) é importante:

Um conteúdo de saber que tenha sido definido como saber a ensinar, sofre, a partir de então, um conjunto de transformações adaptativas que irão torná-lo apto a ocupar um lugar entre os objetos de ensino. O 'trabalho' que faz de um objeto de saber a ensinar, um objeto de ensino, é chamado de transposição didática. (Chevallard, 1986, p.90).

As dificuldades impostas pelos exames vestibulares são inúmeras no mundo da concorrência por uma vaga. Atualmente o Exame Nacional do Ensino Médio, ENEM, propõe um tempo de cerca de três minutos para resolução de questões propostas a um item. A dificuldade vai muito além das habilidades em saber o conteúdo correto; é necessária muita concentração, rapidez nos cálculos e interpretação racional das questões para que se possa resolver em apenas três minutos o item.

O currículo da educação básica, particularmente o do ensino médio é regido pelos Parâmetros Curriculares Nacionais (PCN, Ensino Médio, 2002), com base nos princípios da Lei de Diretrizes e Bases da Educação Nacional (9.394/96, LDB). A esse respeito, descreve o artigo 22 que a educação básica tem por finalidades desenvolver o educando, assegurar-lhe formação comum indispensável para o exercício da cidadania e fornecer meios para progredir no trabalho e em estudos posteriores (Brasil, 1996, p. 22).

A nova Base Nacional Comum Curricular estabelece as disciplinas Língua Portuguesa e Matemática como obrigatórias, e as demais estão inseridas nas áreas de conhecimento, de maneira interdisciplinar, que deverão ser escolhidas pelos alunos (competências específicas para cada área de conhecimento).

Apesar desta inovação, Pinto (2017) faz um questionamento acerca do papel da disciplina Matemática no ensino aprendizagem, especialmente no ensino médio e cita a questão curricular:

Essa nova configuração, dando destaque à Matemática, nos leva a indagar sobre o papel que essa disciplina escolar representa no desenvolvimento dos jovens. Esse destaque atribuído à Matemática evidencia a necessidade de problematizar o seu ensino e aprendizagem, indagando sobre o porquê da inclusão ou exclusão deste ou daquele conteúdo (Pinto, 2017, p. 1048).

Muitas serão as questões a serem discutidas, sem dúvidas, porém, quanto ao ensino da matemática, cerne deste artigo, no trabalho com os números devem-se valorizar questões relacionadas à geometria plana e espacial, possibilitando ao estudante compreender aspectos simples e visuais que dispendam muito trabalho simplificando seus cálculos.

\section{Demonstração da Relação das Infinitas Ternas Pitagóricas com a Hipérbole}

Porém, uma das funções exponenciais não elementares mais conhecidas, como a função exponencial com sinal negativo no expoente, encontrada em uma etapa importante na resolução da Curva de Erro Normal atribuída ao matemático francês Abraham de Moivre, este artigo propõe resolver uma função semelhante. 
Segundo Boyer, (2003) e Eves (2011), foi levado em consideração o trabalho de Carl Friedrich Gauss sobre o uso de quadrados perfeitos. Mesmo assim, o método que Gauss utiliza é, por meio da mudança de coordenadas, e em um domínio diferente do exposto neste artigo. Existe outro método, que leva em consideração algumas manipulações com a função Gama, que pode ser convenientemente utilizado para resolver o mesmo problema atacado por Gauss, embora seja mais trabalhoso.

De acordo com Joseph, G. G. (1991),

Teorema 2.1 Todo número quadrado perfeito pode ser representado pela igualdade 1.

$$
n^{2}=(2 n-1)+(n-1)^{2}
$$

Prova direta do teorema (1)

$$
\begin{gathered}
n^{2}=(2 n-1)+(n-1)^{2} \\
n^{2}=2 n-1+n^{2}-2 n+1 \\
n^{2}=n^{2}=n^{2}
\end{gathered}
$$

Segundo Scheinerman, E. R. (2016), tem-se:

Teorema 2.2 Todo número quadrado perfeito $\mathrm{x}^{2}$ pode ser representado por uma terna pitagórica, desde que $\mathrm{x}$ seja ímpar.

$$
\left(\frac{x^{2}+1}{2}\right)^{2}=x^{2}+\left(\frac{x^{2}-1}{2}\right)^{2}
$$

Teorema 2.3 Todo número quadrado perfeito $\mathrm{x}^{2}$ pode ser representado por uma terna pitagórica, desde que $\mathrm{x} \in \mathrm{R}$.

$$
\left(x^{2}+1\right)^{2}=4 x^{2}+\left(x^{2}-1\right)^{2}
$$

Prova do teorema (2) Como:

$$
n^{2}=(2 n-1)+(n-1)^{2}
$$

Segue que:

$$
2 n-1=x^{2} \rightarrow n=\frac{x^{2}+1}{2} \text { e } n-1=\frac{x^{2}-1}{2}
$$

Logo,

$$
n^{2}=(2 n-1)+(n-1)^{2} \rightarrow\left(\frac{x^{2}+1}{2}\right)^{2}=x^{2}+\left(\frac{x^{2}-1}{2}\right)^{2}
$$

Portanto,

$$
\left(\frac{x^{2}+1}{2}\right)^{2}=x^{2}+\left(\frac{x^{2}-1}{2}\right)^{2}
$$

Teorema 2.4 Todo número quadrado perfeito $\mathrm{x}^{2}$ pode ser representado por uma terna pitagórica, desde que $\mathrm{x} \in \mathrm{R}$.

$$
\left(x^{2}+1\right)^{2}=4 x^{2}+\left(x^{2}-1\right)^{2}
$$

\section{Relação das Ternas Pitagóricas com uma Hipérbole Equilátera}

Na concepção de Lehmann, C. H. (1998), tem-se:

Teorema 3.1. Todo número real pode ser representado por uma hipérbole equilátera.

$$
y^{2}-z^{2}=1
$$

Prova do teorema (3.1)

$$
x^{2}=\left(\frac{x^{2}+1}{2}\right)^{2}-\left(\frac{x^{2}-1}{2}\right)^{2}
$$

Logo, rearranjando a expressão acima, tem se que, 


$$
1=\left(\frac{x^{2}+1}{2 x}\right)^{2}-\left(\frac{x^{2}-1}{2 x}\right)^{2}
$$

Portanto,

$$
\left(\frac{x^{2}+1}{2 x}\right)^{2}-\left(\frac{x^{2}-1}{2 x}\right)^{2}=1
$$

Ao se fazer,

$$
y=\left(\frac{x^{2}+1}{2 x}\right)^{2} \text { e } z=\left(\frac{x^{2}-1}{2 x}\right)^{2}
$$

Tem se que, podemos obter a representação de uma hipérbole equilátera conhecida e demonstrada por, Nóbrega (2014); Joseph (1991); Simmons (2009) a seguinte relação como segue:

$$
y^{2}-z^{2}=1
$$

Que é uma hipérbole equilátera.

\section{Metodologia}

Esta pesquisa é classificada como quantitativa. Escolhemos este modelo de pesquisa porque mostra, de forma simples, os fatores e aspectos relevantes e atuais da disciplina de Cálculo no meio educacional brasileiro, bem como seu impacto no meio científico matemático.

Este trabalho é uma investigação de diagnóstico e abordagem (quantitativa), O estudo envolveu uma abordagem quantitativa que utiliza métodos quantitativos, Pereira, et al. (2018), foi realizada considerando o período de 01 de maio de 2020 a 20 de março de 2021 de caráter exploratório.

As relações derivadas do teorema de Pitágoras são muito utilizadas na geometria plana e espacial, além de outras aplicações na geometria diferencial. O fato de se conhecer os principais ternos pitagóricos pode ser determinante na resolução de exercícios e questões do ENEM, Exame Nacional do Ensino Médio, como também em concursos públicos, onde o tempo de resolução e descoberta é primordial no desenvolvimento e conclusão destes exames, onde a classificação tem relevância direta na vida do candidato.

Durante a execução da aula, muitos professores de matemática ensinam a demonstração das fórmulas e teorias para depois realizar alguns exercícios a fim de práticas todas as relações encontradas. Muitas vezes essa metodologia de deduzir e demonstrar as fórmulas e ralações matemáticas não é a mais adequada para o tempo em que vivemos.

$\mathrm{Na}$ atualidade temos um momento de total dicotomia no ensino da matemática aos alunos. De um lados as demonstrações e resolver as questões sem usar qualquer tipo de fórmula ou relação, ou seja, resolver a partir dos princípios básicos teóricos ensinados até chegar ao resultado, ou então, usar fórmulas ou transposições didáticas que levam ao resultado rapidamente.

No ensino da matemática ainda não encontramos um método adequado aos exames de massa, em que compartilhe da dedução e da transposição sem prejuízo ao ensino. O que provavelmente falte é fazer mais exercícios práticos e usuais destes exames de massa, criando no aluno uma certa flexibilidade e confiança de saber resolver e não deixá-lo entregue aos lobos, ou seja só terá conhecimento no momento em que for fazer o exame o que certamente será demasiado tardio.

Relações que aparecem muito no cotidiano, observe a Figura 1: 
Figura 1: Aplicações de múltiplos do triângulo retângulo.

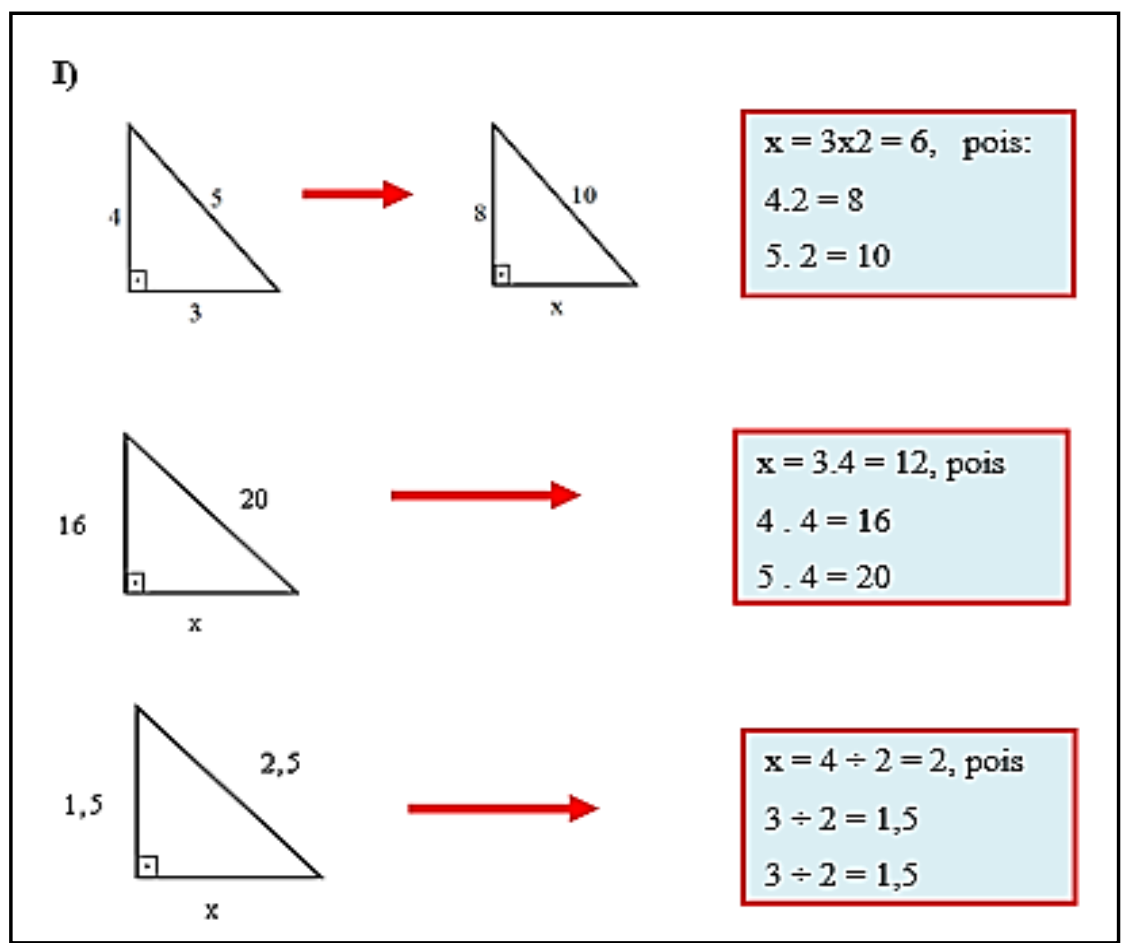

Fonte: Dias, G. N. (2011).

Na Figura 1, observamos a ocorrência dos lados múltiplos do triângulo retângulo 3,4 e 5 e como devemos fazer para descobrir os demais lados.

Também o caso da Figura 2, abaixo é muito recorrente:

Figura 2: Aplicações de múltiplos do triângulo retângulo.

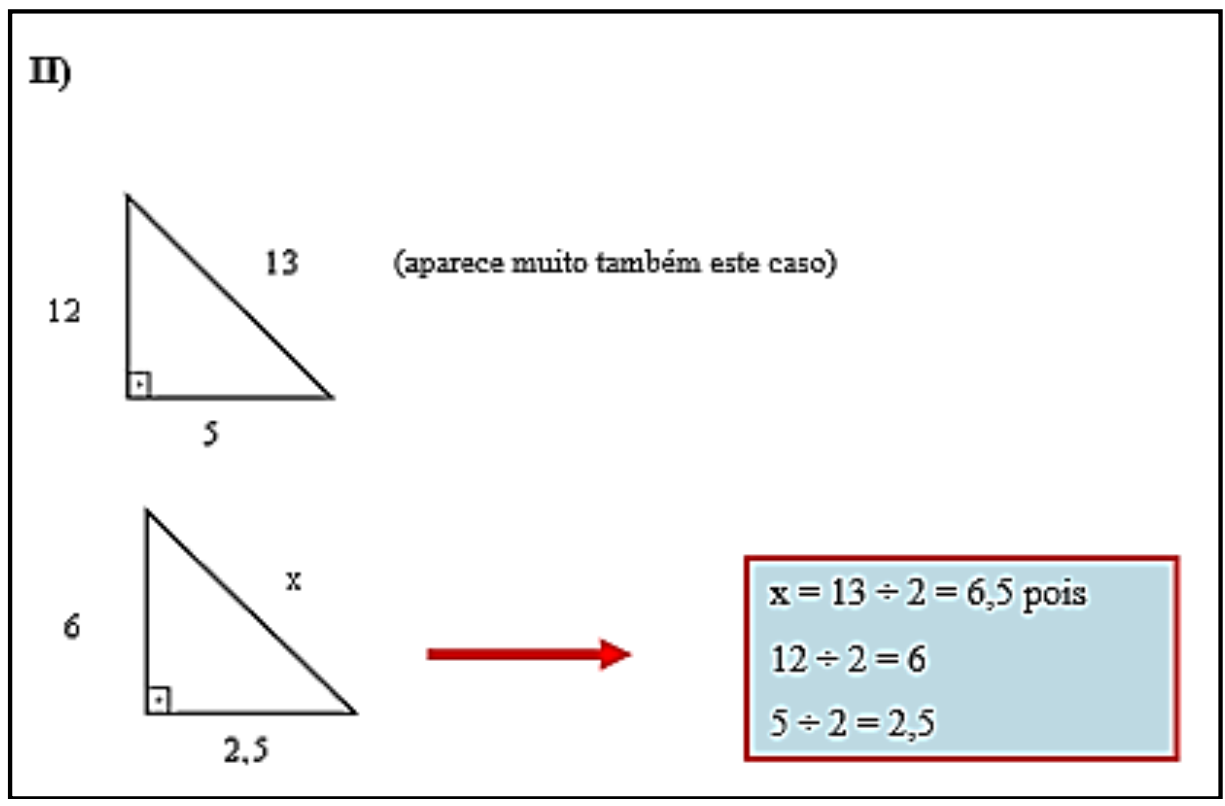

Fonte: Dias, G. N. (2011).

Na Figura 2, mostra outro caso recorrente de triângulo retângulo pitagórico, muito utilizado em exames de massa, reforçando a ideia central da Figura 1.

Existem algumas generalizações do Triângulo retângulo; chamados de termos Pitagóricas, segue casos principais, Figura 3: 
Figura 3: Tipo 01, ternas pitagóricas.

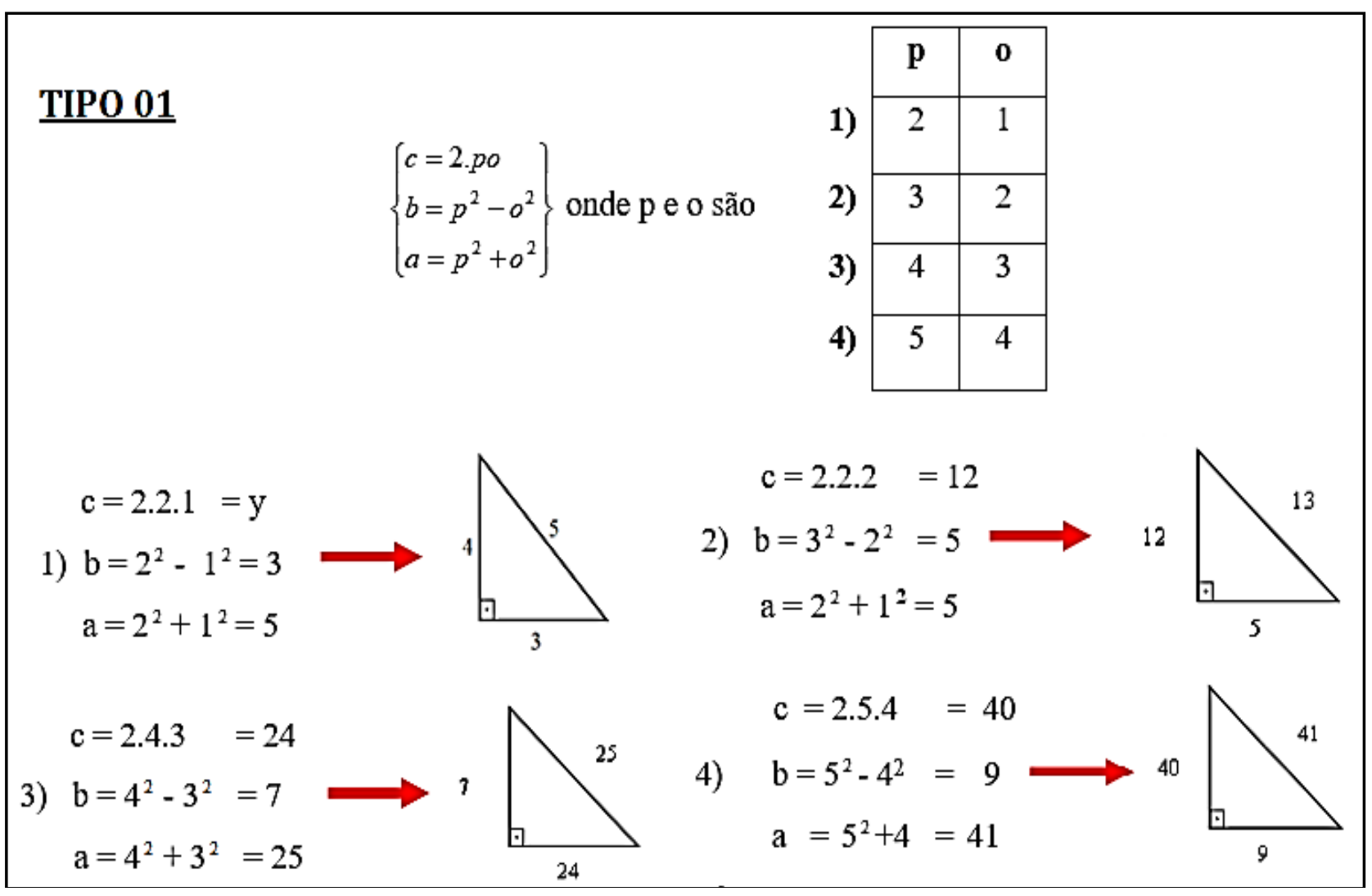

Fonte: Alencar Filho (1987).

Na Figura 3, é mostrado o tipo 1, onde podemos descobrir inúmeros triângulos pitagóricos, utilizando a relação descrita como a base para obtermos mais tipos de triângulos pitagóricos, quando for preciso. É mais usada por professores ao elaborarem questões de geometria plana e Espacial e precisão de vários tipos de triângulo retângulo.

Segue mais um tipo de terno Pitagórico e mais utilizado no cotidiano, Figura 4: 
Figura 4: Tipo 02, ternas pitagóricas.

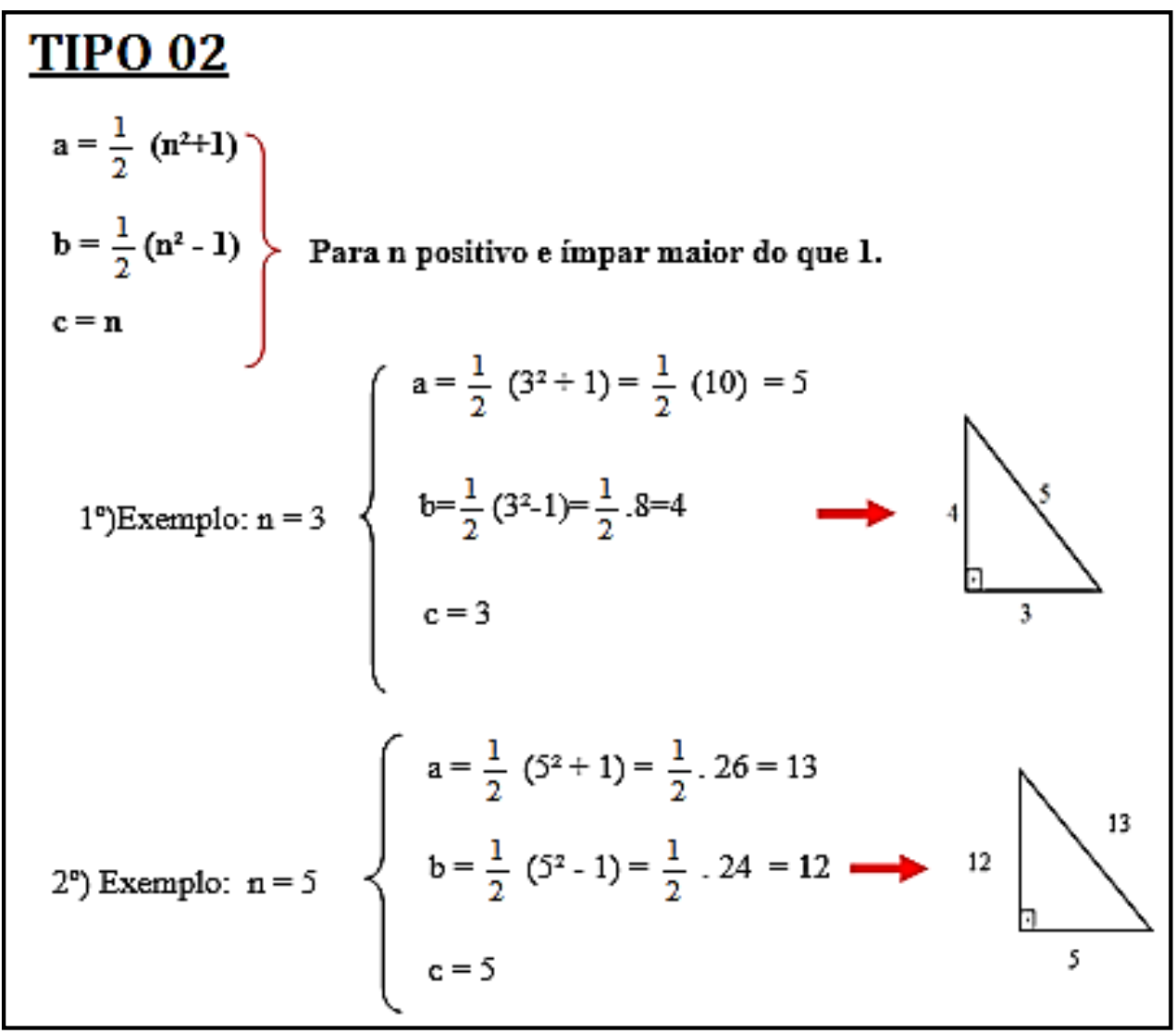

Fonte: Alencar Filho (1987).

Na Figura 4, é mostrado o tipo 2, onde podemos descobrir inúmeros triângulos pitagóricos, utilizando a relação descrita como a base para obtermos mais tipos de triângulos pitagóricos, quando for preciso. É mais usada, também, por professores ao elaborarem questões de Geometria Plana e Espacial e a fim de descobrir vários tipos de triângulo retângulo. O tipo 2 é o que apresenta menor dificuldade para memorização e utilização, pelo fato de apresentar apenas uma variável inicial a ser considerada, sempre iniciando por um número ímpar n.

\section{Resultados e Discussões}

A forma realizada para apresentar resultados, foi selecionando alguns casos de aplicação das ternas em questões usuais no Exame Nacional do Ensino Médio e em concursos militares, momento em que a característica geral dessas provas é a quantidade grande de questões de matemática e outras disciplinas e o pequeno tempo para resolvê-las.

Exemplo de aplicação na geometria plana

Segue Figura 5, mostrando a aplicação: 
Figura 5: Questão do ENEM.

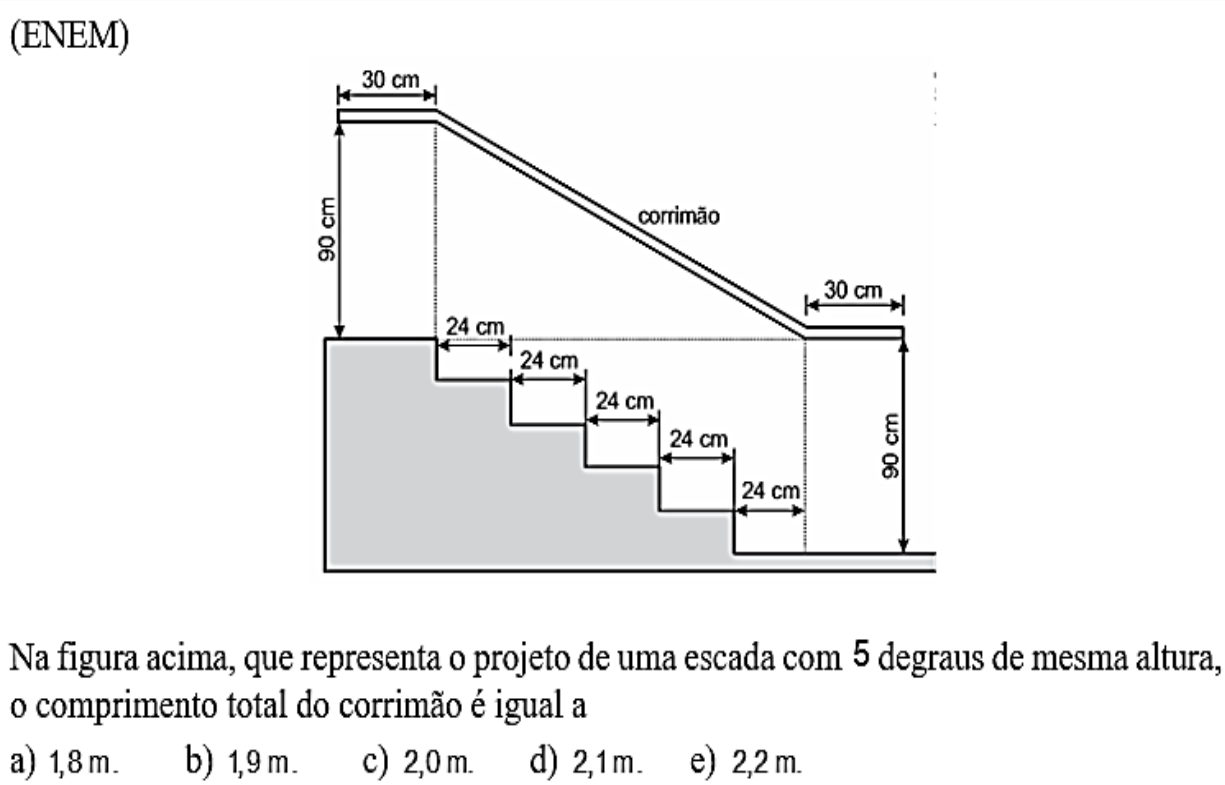

Fonte: Dias, G.N. et al (2021).

$\mathrm{Na}$ Figura 5, é amostrada uma questão do Exame Nacional do Ensino Médio, onde se verifica a necessidade da utilização dos conceitos de Ternos Pitagóricos, justamente para ser ter a diminuição do tempo de resolução e redução do risco de erro em cálculos elementares, muito comum nos exames de massa.

Segue a resolução proposta, Figura 6:

Figura 6: Resolução da questão proposta sem e com o uso das ternas Pitagóricas.

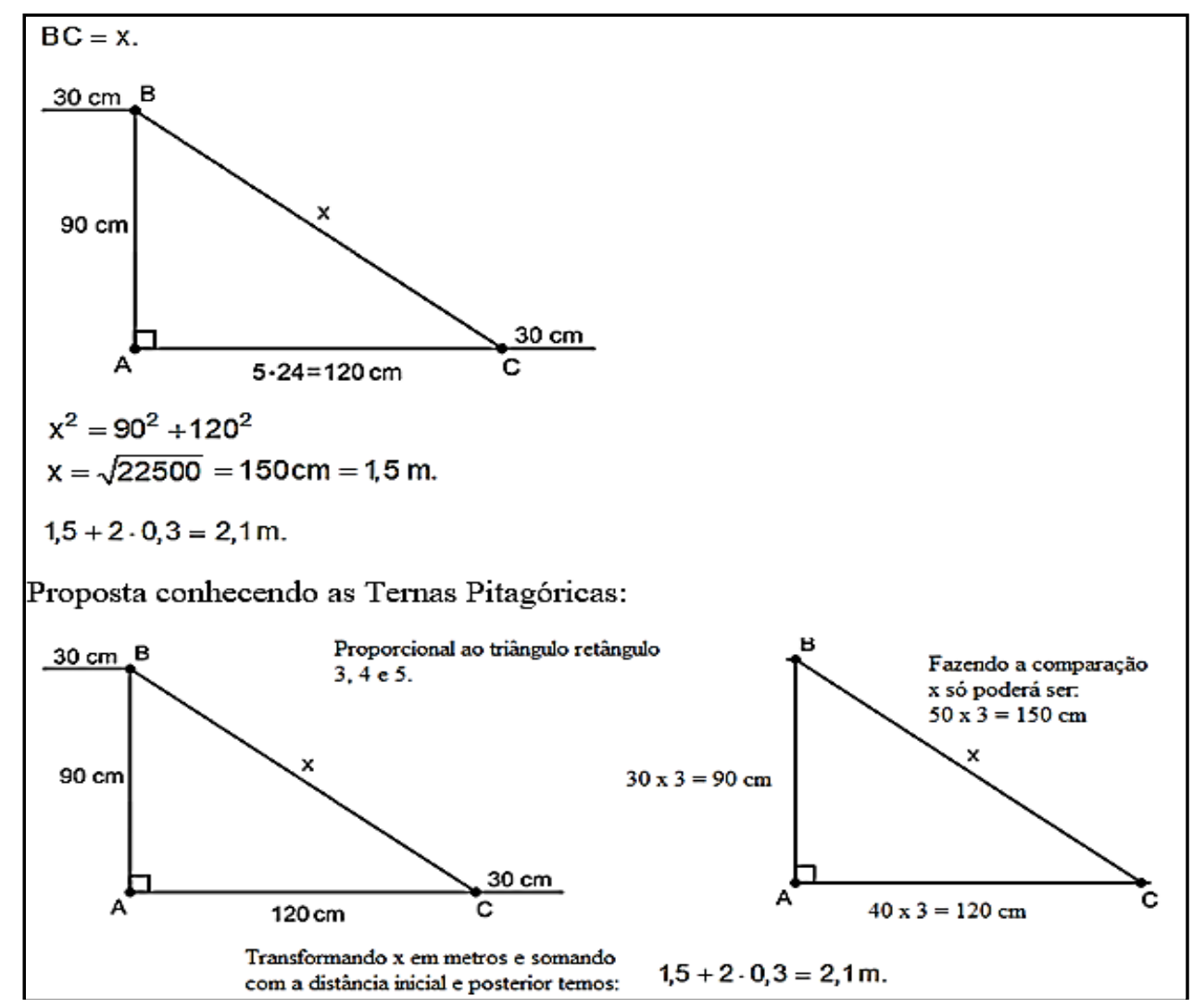

Fonte: Dias, G. N. (2011). 
Observando a solução da figura 6, aparentemente sem o uso de ternas, ao aluno que se preparou adequadamente, provavelmente vai perder tempo elevando ao quadrado 90 e 120 e depois fatorando para extrair da raiz. Com a utilização das ternas Pitagóricas como uma forma de transposição didática terá um avanço no tempo de resolução que é determinante na prova do ENEM.

Exemplo de aplicação na geometria Espacial

Segue Figura 7, exemplo de aplicação:

Figura 7: Aplicação dos ternos Pitagóricos na Geometria Espacial.

(ITA) Uma esfera está inscrita em uma pirâmide regular hexagonal cuja altura mede $12 \mathrm{~cm}$ e a aresta da base mede $\frac{10}{3} \sqrt{3} \mathrm{~cm}$. Então o raio da esfera, em cm, é igual a
a) $\frac{10}{3} \sqrt{3}$.
b) $\frac{13}{3}$
c) $\frac{15}{4}$.
d) $2 \sqrt{3}$.
e) $\frac{10}{3}$.

Fonte: Dias, G. N. (2011).

Na Figura 7, observamos que o uso de ternos pitagóricos é utilizado também para questões de provas de concursos militares, ratificando a ideia da importância e relevância da sua utilização.

Segue Figura 8, aplicação dos ternos pitagóricos na Geometria Espacial:

Figura 8: Resolução da questão utilizando ternos pitagóricos.

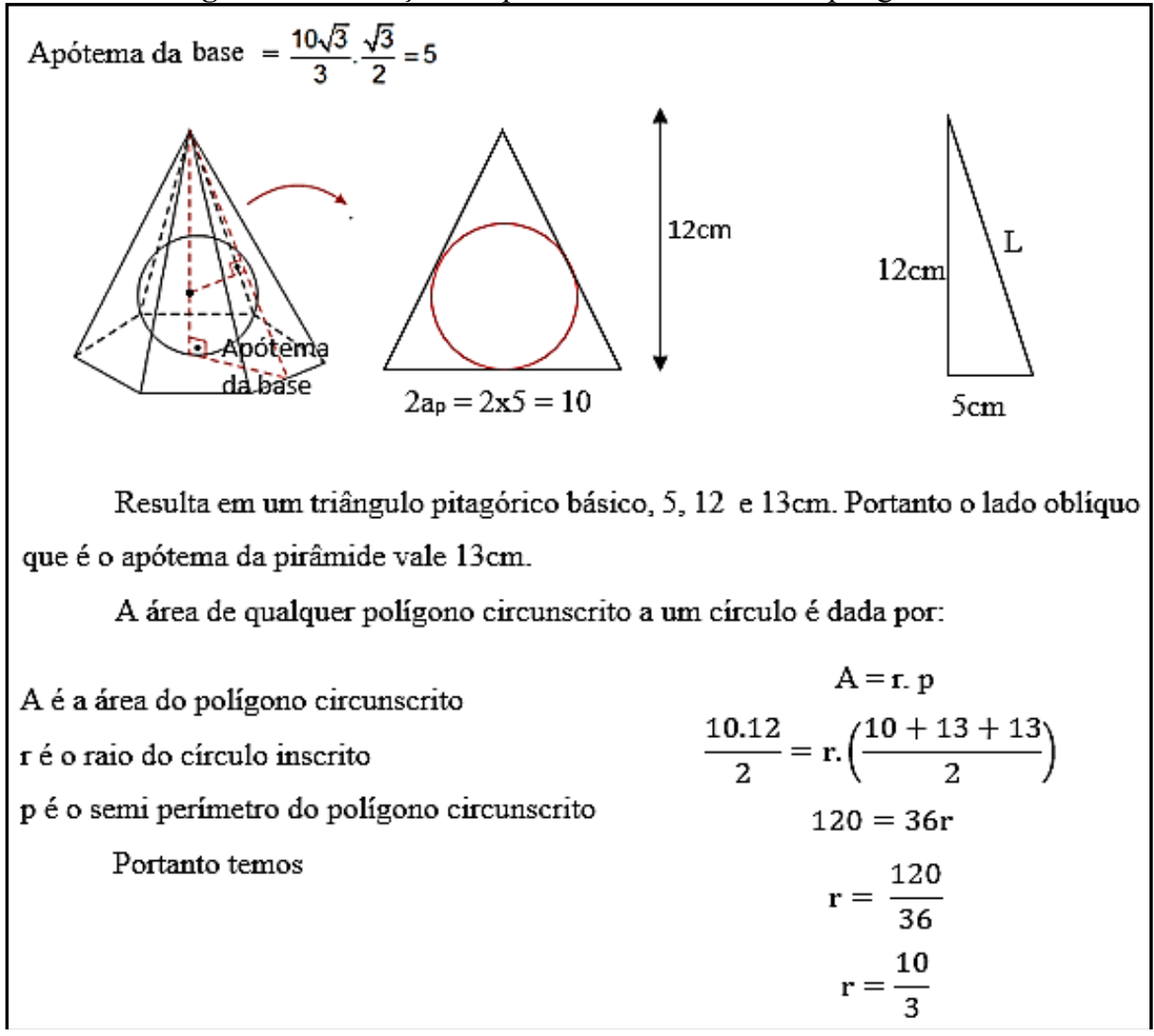

Fonte: Dias, G. N. (2011).

Na Figura 8, observamos a simplicidade da solução das questões de concursos militares na utilização dos ternos pitagóricos. 


\section{Considerações Finais}

O tema Ternas Pitagóricas, traz um conteúdo de extrema relevância ao Ensino Médio e ao Ensino Fundamental II, por mostrar uma recorrência comum que aparece com frequência na resolução de exercícios de Geometria Plana e Espacial, facilitando e tornando mais rápida e ágil a solução, bastando achar o triângulo retângulo base e a partir daí calcular o seu lado múltiplo, sem necessidade de utilizar o teorema de Pitágoras.

A utilização das ternas pitagóricas favorece a agilidade nos cálculos geométricos e trigonométricos, uma vez que os lados poderão ser múltiplos do triângulo retângulo base. Além do que nas questões de vestibulares e também do Exame Nacional do Ensino Médio, geralmente os valores são exatos para não confundir o candidato, uma vez que o mesmo não pode usar calculadora, por esta razão há necessidade dos resultados ser números exatos, o que favorece a utilização das ternas pitagóricas na geometria plana e espacial, fazendo o diferencial em se tratando de tempo de resolução da questão, o que é importante ao candidato naquele momento e para a sua classificação e aprovação.

Nos exames de ingresso nas universidades, o tempo tem sido o principal adversário do candidato, principalmente na matemática, no Exame Nacional do Ensino Médio a Matemática ocupa 45 questões, respondendo por 25\% das questões objetivas, representando $1 / 4$ da nota do aluno, onde se tem em torno de 3 minutos para responder cada questão, o que nem sempre apresenta o tempo estimado que muitas vezes diverge do real.

\section{Sugestões Para Trabalhos Futuros}

O trabalho a seguir serve de base para futuros trabalhos, principalmente no desenvolvimento das aulas de geometria plana e espacial, sendo os conceitos aqui tratados são necessários ao pré-requisito ao início destas unidades curriculares, pois o seu uso é recorrente em todo o andamento pedagógico desta unidade facilitando a compreensão e raciocínio lógico a ser utilizado e dessa forma poderíamos evoluir no ensino de jovens e adultos investigando essas situações de um olhar mais próximo ao da atualidade.

\section{Referências}

Alencar Filho, Edgard de (1987): Teoria Elementar dos Números, (2a ed.), Nobel

Brasil. (1996). Lei $n^{\circ} 9.394$ de 20 de dezembro de 1996. Estabelece as diretrizes e bases da educação nacional http://www.planalto.gov.br/ccivil_03/LEIS/L9394.htm

Brasil (1996). Lei de Diretrizes e Bases da Educação. MEC. Ministério da Educação. Secretaria de Educação Básica e Tecnologia.

Brasil. (2002). Parâmetros Curriculares Nacionais + Ensino Médio: Orientações Educacionais complementares aos Parâmetros Curriculares Nacionais: Ciências da Natureza, matemática e suas Tecnologias. Secretaria de Educação Média e Tecnológica. MEC, SEMTEC.

Brasil. (2017). Base Nacional Comum + Ensino Médio: Documento homologado pela Portaria n 1.570 , publicada no D.O.U. de 21/12/2017, Seção 1, Pág. 146.

Boyer, C. B. (2003). História da Matemática. São Paulo: Edgard Bencher Ltda.

Chevallard, Y. (1986). La transposition didactique: du savoir savant au savoir enseigné. Ed. Colomb Jacques. p. 89-91.

Dias, G. N. (2011) Práticas do Ensino da Matemática: a realidade da sala de aula / Gustavo Nogueira Dias. - Belém, Pará.

Dias, G. N., Barreto, W. D. L. \& Vogado, G. E. R. (2020) Matemática Aos Futuros Militares, Gustavo Nogueira Dias. - Belém, Pará. https://doi.org/10.29327/530351

Dias, G. N., Vogado, G. E. R. \& Silva, P. R. S. Da (2021). Matemática Interativa e Descritiva-Belém. Pará https://doi.org/10.29327/530352

Eves, H. (2011). Introdução à história da matemática / Howard Eves, tradução Hygino H. Domingues. (5a ed.), Editora da Unicamp.

Iezzi, Gelson (1993). Fundamentos da Matemática Elementar, 4, Ed. Atual.

Joseph, G. G. (1991). The crest of the peacock: Non-European roots of mathematics. Princeton, NJ: Princeton University Press. 
Research, Society and Development, v. 10, n. 13, e281101321391, 2021

(CC BY 4.0) | ISSN 2525-3409 | DOI: http://dx.doi.org/10.33448/rsd-v10i13.21391

Lehmann, C. H. (1998), Geometria Analitica, (9a ed.), Ed. Globo.

Maev Kennedy (2017) Mathematical secrets unlocked after nearly a century of study. Jorunal The Guardian Live, 24.08.2017.

Nóbrega, J. A. da. (2014). Simulação numérica de distribuição de temperaturas e tensões residuais em juntas soldadas de aço API 5 L X80 pelos processos de soldagem GTAW e SMAW / Dissertação (Mestrado em Engenharia Mecânica) - Universidade Federal de Campina Grande, Centro de Ciências e Tecnologia.

Pereira, A. S., et al. (2018). Metodologia da pesquisa cientifica. UFSM.

Pinto, A. H. (2017). A Base Nacional Comum Curricular e o Ensino de Matemática: flexibilização ou engessamento do currículo escolar. Bolema, (SP), 31(59), 1045-1060.

Scheinerman, E. R. (2016). Matemática Discreta: Uma Introdução, Cengage Leranig.

Secco, L. C. M., Cabral, N. F., Chaquiam, M., Dias, G. N., Pamplona, V. M. S., Reis, C. P., Costa, E. G. \& Pinto, G. P. (2020) O ensino de juros compostos por meio de sequências didáticas. Research, Society And Development, 9, e17691211068.

Simmons, G. F. (2009). Cálculo com Geometria Analítica, 2, McGraw-Hill. 